\title{
Liver transplantation for hepatocellular carcinoma with live donors or extended criteria donors: a propensity score-matched comparison
}

\author{
Georgios C. Sotiropoulos ${ }^{\mathrm{a}, \mathrm{b}}$, Eleftherios Spartalis ${ }^{\mathrm{b}}$, Nikolaos Machairas ${ }^{\mathrm{b}}$, Andreas Paula, \\ Massimo Malagóa,c, Markus Neuhäuser ${ }^{d}$ \\ Essen University Hospital, Germany; National and Kapodistrian University of Athens, Greece; Royal Free Hospital, \\ University College London, UK; Koblenz University of Applied Science, Remagen, Germany
}

\section{Abstract}

${ }^{a}$ Department of General, Visceral and Transplantation Surgery, Essen University Hospital, Essen, Germany (Georgios C. Sotiropoulos, Andreas Paul, Massimo Malagó); becond Department of Propaedeutic Surgery, National and Kapodistrian University of Athens, Athens, Greece (Georgios C. Sotiropoulos, Eleftherios Spartalis, Nikolaos Machairas); 'Department of HPB and Liver Transplant Surgery, Royal Free Hospital, University College London, London, UK (Massimo Malago); ${ }^{\mathrm{d} D e p a r t m e n t ~ o f ~ M a t h e m a t i c s ~ a n d ~ T e c h n o l o g y, ~ K o b l e n z ~}$ University of Applied Science, Remagen, Germany (Markus Neuhäuser)

Conflict of Interest: None

Correspondence to: Prof. Dr. Georgios C. Sotiropoulos, MD, PhD, FACS, FEBS, Department of General, Visceral and Transplantation Surgery, Essen University Hospital, Hufelandstr. 55, 45122 Essen, Germany, e-mail: georgios.sotiropoulos@uni-due.de

Received 6 February 2018; accepted 29 June 2018; published online 27 July 2018

DOI: https://doi.org/10.20524/aog.2018.0301

\section{Introduction}

Hepatocellular carcinoma (HCC) is one of the most common malignancies worldwide, accounting for about 1 million related deaths annually. Its incidence is estimated to have increased by $75 \%$ in the United States in the last decade and reflects the increasing prevalence of chronic viral hepatitis [1]. An estimated 372,000 new cases of hepatocellular carcinoma (HCC) are diagnosed each year, constituting $4.6 \%$ of all new human cancers [2]. Liver transplantation (LT) is considered to be the treatment of choice for early HCC in patients with end-stage liver failure, but is limited by donor organ availability. Despite recent criticism, the Milan criteria (single tumor up to $5 \mathrm{~cm} ; 2$ or 3 tumors, none larger than $3 \mathrm{~cm}$; absence of vascular invasion) are most often used when determining organ allocation for HCC patients being considered for deceased donor LT [3]. 
In recent years, live donor LT (LDLT) has become the most likely alternative for the expansion of the organ pool for adult patients with HCC [4-10], but its indications and criteria are still subject to debate [11-16]. Extended criteria donors (ECD) represent another reliable alternative. Very limited information, however, is currently available concerning their comparative outcomes, i.e., in the comparison of transplant outcomes using a reduced (about 50\%) but "high-quality" liver from a live donor or a whole but "low-quality" liver from an extended criteria donor. The aim of this propensity score-matched study was to compare patient survival after LDLT or ECD-LT for HCC.

\section{Patients and methods}

We considered all adult patients who underwent LT in Essen University Hospital, Germany, over a 12 -year period. Data were collected prospectively through both the Eurotransplant database and patient records. Recipients with acuteliver failure, patients listed with high urgency for re-transplantation, or patients receiving split LT were excluded. All operations were performed using standard surgical techniques. All patients received and signed informed consent at the time of listing. Patients with potential live liver donors were evaluated on a case-by-case basis according to their age, severity of liver disease, $a$-fetoprotein (AFP) levels, and HCC characteristics at the time of presentation. A very strict evaluation protocol for the potential donors was applied, resulting in only $14 \%$ suitability in the adult group in our institution [16].

ECD grafts were offered to HCC patients in an effort to expand the donor pool. ECD grafts were defined according to Eurotransplant Foundation rules, as reported elsewhere [17]. Briefly, during the study period, some of the features that shape the profile of an ECD were the following: donor age $>55$ years; Intensive Care Unit (ICU) stay $>5$ days; use of vasopressors; history of cardiopulmonary resuscitation; adiposity (donor body mass index $[\mathrm{BMI}]>25 \mathrm{~kg} / \mathrm{m}^{2}$ ); peak serum sodium $>155 \mathrm{mEq} / \mathrm{L}$; macrovesicular steatosis $>30 \%$; elevated levels of aspartate aminotransferase and/or alanine aminotransferase; cold ischemia time $>10 \mathrm{~h}$; warm ischemia time $>40 \mathrm{~min}$; and risk of disease transmission from the donor to the recipient in the case of: a) viral hepatitis $\mathrm{B}$ or $\mathrm{C}$ infection; b) sepsis, bacteremia, meningitis; c) history of malignancy; or d) drug abuse. This retrospective, single-center, cohort study was approved by the local ethics committee and conformed to the 1975 Declaration of Helsinki.

All patients were examined using spiral computed tomography technology and intravenous contrast material. Abdominal ultrasonography and bone scintigraphy were additionally performed in all patients. Serial AFP levels were obtained prior to and after LT. The diagnosis of HCC was established according to the consensus statement from the European Association for the Study of the Liver. The clinical classification was based on the morphological description of the tumor according to the radiological findings of 2 independent radiologists.

The following recipient characteristics were evaluated: age, sex, etiology of liver disease, laboratory model for end-stage liver disease (labMELD) score, HCC-specific bridging treatment (radiofrequency ablation or transarterial chemoembolization in most cases), and conformance to the Milan criteria. All explanted livers were examined micro- and macroscopically by an experienced pathologist. The tumor number and size, the occurrence of tumor satellites, the presence of vascular invasion or tumor thrombi, the resection margin, the tumor differentiation, and the classification according to the Milan criteria, were documented. Follow-up data included the need for re-transplantation (Re-LT), the occurrence of ischemictype biliary lesions (ITBL), the current patient status, recurrent disease and the treatment of recurrence, and the cause of death.

Follow-up studies included CT scans of the abdomen and chest, and measurement of AFP levels every 4 months during the first year after transplantation, every 6 months during the second year, and yearly thereafter. Minimum follow up was either 5 years or until death. No patient was lost to follow up.

Patient survival was our primary outcome. For the secondary outcomes, three major concerns associated with the application of both LD and ECD grafts were explored: 1) rates of Re-LT, which may be the result of small-for-size syndrome after LDLT, or of primary non-function after ECD-LT; 2) rates of ITBL, described as complications in the follow up of both procedures; and 3) tumor recurrence, given that in the setting of LDLT or ECD-LT the Milan criteria are not always strictly followed.

\section{Statistical analysis}

Continuous data were expressed as median and range and compared using Student's $t$-test. Categorical data were compared using Fisher's exact test. The primary outcome was statistically analyzed using Kaplan-Meier estimates and Cox proportional hazards regression. Logistic regression analyses were used for statistical analysis of the secondary outcomes. Propensity score was calculated based on patient age, sex, hepatitis $C$ viral infection (HCV), labMELD score, bridging treatment, Milan criteria, AFP levels, and tumor grade. Differences with $\mathrm{P}<0.05$ were considered to be statistically significant. Statistical analyses were performed using SAS (SAS Institute).

\section{Results}

We reviewed data on 109 consecutive LT for HCC performed in Essen University Hospital, Germany, and which met the inclusion criteria. Of these, 57 used LD grafts and 52 ECD grafts. Patient characteristics and follow-up data are shown in Table 1. Median recipient age was 56 (range 18-69) years. The majority were male $(\mathrm{n}=79,72 \%)$. the main cause of chronic liver disease was HCVinduced liver cirrhosis $(n=42,39 \%)$. Half the patients $(n=55)$ had undergone transarterial chemoembolization or radiofrequency ablation as HCC-specific bridging treatment prior to LT. Fifty-six patients met the Milan criteria at the time of listing. The median value of AFP was $21 \mathrm{ng} / \mathrm{mL}$. Vascular invasion was documented in 21 liver explants (19\%). Tumor differentiation was assessed as G1 ( $n=18)$, G2 ( $n=59), G 3(n=13)$, or $\mathrm{Gx}(\mathrm{n}=10)$. In 9 instances no living tumor cells were found on pathological examination, 
as a consequence of $100 \%$ tumor necrosis after successful bridging treatment. Urgent Re-LT with a deceased donor graft was required in 15 cases, in 8 of them following an LDLT. ITBL was documented in 18 patients, of whom the majority $(n=11)$ underwent ECD-LT. Twenty-four patients experienced a post-LT tumor recurrence, 14 after ECD-LT and 10 after LDLT. At the time of the statistical analysis for this study, 73 patients were alive after a median follow up of 36 months. The causes of death in each group are shown` in Table 2.

\section{Extended criteria donors}

Donors were classified as ECD according to the following criteria: donor age $>55$ years, 25 patients; ICU stay $>5$ days, 20 patients; use of vasopressors, 44 patients; donor BMI $>25 \mathrm{~kg} / \mathrm{m}^{2}, 29$ patients; peak serum sodium $>155 \mathrm{mEq} / \mathrm{L}$, 19 patients; macrovesicular steatosis $>30 \%$, 4 patients; elevated

Table 1 Patients' characteristics and follow-up data

\begin{tabular}{lcc}
\hline Variable & LDLT $\mathrm{n}=57$ & ECD-LT $\mathrm{n}=52$ \\
\hline Recipient age (median, range) & $55(18-67)$ & $57(29-70)$ \\
Sex (male) & 42 & 37 \\
\hline Hepatitis-related cirrhosis & 35 & 32 \\
\hline HCV infection & 23 & 19 \\
LabMELD & 10 & 12 \\
Bridging treatment (yes) & 22 & 33 \\
\hline Milan criteria satisfied & 24 & 32 \\
AFP (ng/mL) & 26 & 17 \\
Tumor grade moderate/poor & $27 / 7$ & $32 / 6$ \\
\hline Patients alive in follow up & 27 & 37 \\
\hline Re-LT & 8 & 7 \\
\hline ITBL & 7 & 11 \\
\hline Tumor recurrence & 10 & 14 \\
\hline
\end{tabular}

LDLT, live donor liver transplantation; ECD-LT, extended criteria donor-liver transplantation; $H C V$, hepatitis $C$ virus; MELD, model for end-stage liver disease; AFP, $\alpha$-fetoprotein; re-LT, repeat liver transplantation; ITBL, ischemic type bile lesions

Table 2 Causes of death in each group

\begin{tabular}{lcc}
\hline Cause of death & LDLT & ECD-LT \\
\hline Primary non-function & 0 & 2 \\
Multi-organ failure & 1 & 3 \\
Pneumonia & 2 & 2 \\
Sepsis & 3 & 3 \\
Graft-versus-host disease & 0 & 1 \\
HCC recurrence & 5 & 1 \\
Small for size & 6 & 0 \\
Pulmonary embolism & 4 & 0 \\
Other & 3 & 0 \\
Total & 24 & 12 \\
\hline
\end{tabular}

LDLT, live donor liver transplantation; ECD-LT, extended criteria donor-liver transplantation; HCC, hepatocellular carcinoma levels of aspartate aminotransferase, 21 patients; alanine aminotransferase, 15 patients; cold ischemia time $>10 \mathrm{~h}$, 9 patients; and warm ischemia time $>40 \mathrm{~min}, 11$ patients.

\section{Primary outcome}

The results of the Cox proportional hazards regression analysis for patient survival are given in Table 3. LT-procedure (hazard ratio [HR] 2.349, 95\%Cls 1.151 to $4.794, \mathrm{P}=0.019$ ), recipient age (HR $1.075,95 \% \mathrm{Cls} 1.020$ to $1.13, \mathrm{P}=0.0074$ ) and labMELD-score (HR 1.082, 95\%Cls 1.021 to $1.147, \mathrm{P}=0.0075$ ) gained statistical significance. After adjustment with the propensity score (stratification with 5 strata, calculated based on age, sex, HCV, labMELD, bridging-treatment, Milan criteria, AFP and tumor grade), the LT procedure was still significant (HR 2.401, $\mathrm{P}=0.0253$; Table 4). As shown in the Kaplan-Meier survival distribution function, LT for HCC showed superior patient survival with ECD grafts rather than LD grafts (Fig. 1).

\section{Secondary outcomes}

The results of the logistic regression analysis for Re-LT, ITBL, and tumor recurrence are presented in Tables 5a, 5b, and $5 c$, respectively. Tumor grade gained statistical significance for Re-LT (odds ratio [OR] 9.628, $\mathrm{P}=0.0391$ ), labMELD score for ITBL (OR 1.224, $\mathrm{P}=0.0306$ ), and Milan criteria for tumor recurrence (OR 6.375, $\mathrm{P}=0.0267$ ), respectively. However, no statistically significant differences were observed between the two transplant procedures (ECD/LDLT).

\section{Discussion}

LT represents the optimal treatment strategy for patients with HCC, as it involves radical oncological resection and Table 3 Results of the Cox regression analysis for survival

\begin{tabular}{lccc}
\hline Variable & $\begin{array}{c}\text { Hazard } \\
\text { ratio }\end{array}$ & $\begin{array}{c}\text { 95\% Confidence } \\
\text { Interval }\end{array}$ & P-value \\
\hline $\begin{array}{l}\text { LT-procedure marginal } \\
\text { graft (reference) }\end{array}$ & & & \\
$\quad$ LT-procedure LDLT & 2.349 & $1.151-4.794$ & 0.0190 \\
Age & 1.075 & $1.020-1.133$ & 0.0074 \\
Sex & 0.752 & $0.338-1.674$ & 0.4857 \\
HCV & 0.609 & $0.318-1.170$ & 0.1364 \\
LabMELD & 1.082 & $1.021-1.147$ & 0.0075 \\
Bridging & 0.536 & $0.258-1.115$ & 0.0950 \\
Milan & 2.115 & $0.847-5.279$ & 0.1086 \\
AFP & 1.000 & $0.99997-1.0002$ & 0.1912 \\
Tumor grade & & & \\
0 (reference) & & & \\
1 & 0.371 & $0.070-1.952$ & 0.2417 \\
2 & 0.930 & $0.297-2.907$ & 0.9003 \\
3 & 1.780 & $0.405-7.819$ & 0.4453 \\
\hline
\end{tabular}

LDLT, live donor liver transplantation; HCV, hepatitis C virus; MELD, model for end-stage liver disease; AFP, $\alpha$-fetoprotein 
Table 4 Results of the stratified Cox regression analysis for survival, the propensity score (calculated based on age, sex, HCV, LabMELD, bridging, Milan, AFP and tumor grade) is the stratification variable, with 5 strata

\begin{tabular}{lccc}
\hline Variable & Hazard ratio & 95\% Confidence interval & P-value \\
\hline $\begin{array}{l}\text { LT-procedure ECD graft } \\
\text { (reference) }\end{array}$ & & & $1.114-5.175$ \\
LT-procedure LDLT & 2.401 & 0.0253 \\
\hline
\end{tabular}

HCV, hepatitis C virus; MELD, model for end-stage liver disease; $A F P$, a-fetoprotein; ECD, extended criteria donor; LDLT, live donor liver transplantation

Table 5 (A) Results of the logistic regression analysis for repeat liver transplantation

\begin{tabular}{lccc}
\hline Variable & Odds ratio & 95\% Confidence interval & P-value \\
\hline LT-procedure ECD graft (reference) & & & $0.111-2.500$ \\
LT-procedure LDLT & 0.527 & $0.944-1.164$ & 0.4204 \\
Age & 1.048 & $0.201-7.571$ & 0.3815 \\
Sex & 1.235 & $0.393-7.955$ & 0.8195 \\
HCV & 1.768 & $0.921-1.155$ & 0.4574 \\
LabMELD & 1.031 & $0.481-13.519$ & 0.5928 \\
Bridging & 2.549 & $0.039-1.448$ & 0.2716 \\
Milan & 0.239 & $0.975-1.005$ & 0.1194 \\
AFP & 0.990 & & 0.0391 \\
Tumor grade $<3$ (reference) & & $1.120-82.752$ & \\
Tumor grade=3 & 9.628 & & \\
\hline ECD, extended criteria donor; LDLT, live donor liver transplantation; HCV, hepatitis C virus; MELD, model for end-stage liver disease; AFP, a-fetoprotein
\end{tabular}

Table 5 (B) Results of the logistic regression analysis for ischemic type bile lesions

\begin{tabular}{lccc}
\hline Variable & Odds ratio & $95 \%$ Confidence interval & P-value \\
\hline LT-procedure ECD graft (reference) & & & $0.824-45.403$ \\
$\quad$ LT-procedure LDLT & 6.116 & $0.943-1.203$ & 0.0766 \\
Age & 1.065 & $0.070-3.569$ & 0.4902 \\
Sex & 0.501 & $0.089-2.678$ & 0.4090 \\
HCV & 0.488 & $1.019-1.471$ & 0.0306 \\
LabMELD & 1.224 & $0.026-1.533$ & 0.1216 \\
Bridging & 0.201 & $0.666-47.224$ & 0.1126 \\
Milan & 5.610 & $0.989-1.005$ & 0.4654 \\
AFP & 0.997 & & 0.5368 \\
Tumor grade $<3$ (reference) & & $0.188-24.715$ & \\
Tumor grade=3 & 2.157 & & \\
\hline
\end{tabular}

ITBL, ischemic type bile lesions; ECD, extended criteria donor; LDLT, live donor liver transplantation; $H C V$, hepatitis $C$ virus; MELD, model for end liver disease; AFP, $\alpha$-fetoprotein; re-LT, re-liver transplantation

improves the underlying liver dysfunction. Currently, there are reported 1-year survival rates of up to $80 \%$, 5-year survival rates up to $70 \%$, and recurrence rates of $10-15 \%$ in patients fulfilling the Milan criteria [18]. The increasing incidence of HCV-related cirrhosis in the Western world during the past 2 decades has led to a corresponding increase in new HCCrelated cases. The potential cohort of new transplant candidates with HCC has grown rapidly. As a result of the scarcity of organs, efforts have focused on expanding the donor pool worldwide, in order to offer LT to more cirrhotic patients [19].

LDLT is an attractive alternative for the expansion of the organ pool for adult patients with HCC and end-stage liver disease [20], and is in part associated with an effort to expand the Milan criteria [21]. In these instances, the strong will to donate among relatives plays a leading role in decision-making [22]. However, the indications for transplantation of HCC patients in the era of LDLT are still being debated. While some centers proposed an expansion of the current listing criteria [4,9], other centers remained conservative [23], emphasizing donor risks as well as a possible return to the suboptimal transplant results of the 1980s. Besides, the overall LDLT setting presupposes an experienced high-volume transplant center and the abovementioned limitations regarding donor safety and long-term oncological results do not allow the wide application of this technique in the western world. 
Table 5 (C) Results of the logistic regression analysis for recurrence

\begin{tabular}{|c|c|c|c|}
\hline Variable & Odds ratio & 95\% Confidence interval & P-value \\
\hline \multicolumn{4}{|c|}{ LT-procedure ECD graftn(reference) } \\
\hline LT-procedure LDLT & 0.966 & $0.294-3.170$ & 0.9545 \\
\hline Age & 1.000 & $0.934-1.072$ & 0.9915 \\
\hline Sex & 0.886 & $0.239-3.278$ & 0.8557 \\
\hline $\mathrm{HCV}$ & 0.556 & $0.178-1.735$ & 0.3121 \\
\hline LabMELD & 1.029 & $0.928-1.141$ & 0.5827 \\
\hline Bridging & 0.391 & $0.114-1.345$ & 0.1364 \\
\hline Milan & 6.375 & $1.239-32.796$ & 0.0267 \\
\hline AFP & 1.000 & $1.000-1.000$ & 0.2309 \\
\hline \multicolumn{4}{|c|}{ Tumor grade $<3$ (reference) } \\
\hline Tumor grade $=3$ & 1.960 & $0.344-11.175$ & 0.4485 \\
\hline
\end{tabular}

ECD, extended criteria donor; $L D L T$, live donor liver transplantation; $H C V$, hepatitis $C$ virus; $M E L D$, model for end-stage liver disease; $A F P, \alpha-f e t o p r o t e i n$

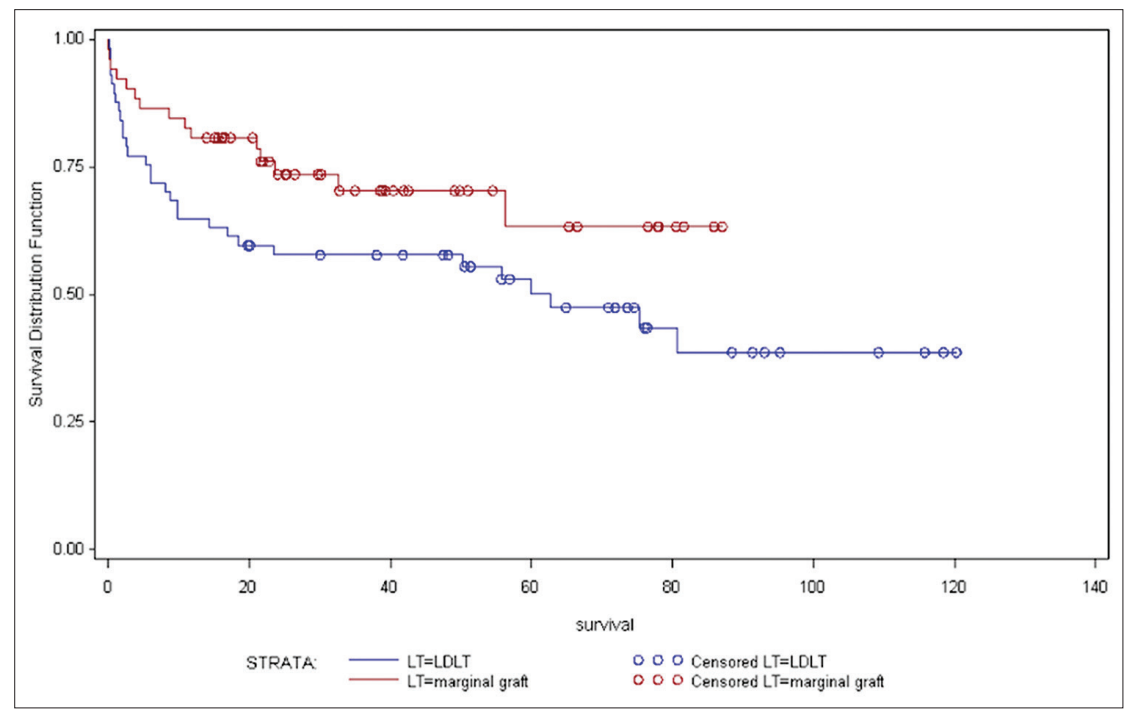

Figure 1 Kaplan-Meier analysis of the two groups

Acceptance of ECD grafts for stable patients with HCC became another potential solution $[14,16,24,25]$. Its application, however, seems possible mostly in large-volume transplant centers and is limited by local allocation rules. A considerable additional limitation of the ECD transplant policy is the quite heterogeneous donor cohort and the diverging definitions between transplant registries, limiting the comparative analysis of published results.

Although in the present study there were no significant differences in Re-LT, ITBL or tumor recurrence rates between the LDLT and ECD groups, LT for HCC showed superior patient survival with ECD rather than LD grafts. We believe this finding can be attributed to the higher rate of "small-forsize" syndrome manifested in patients receiving an LD graft, which seems to confer a survival benefit inferior to that of a theoretically lower quality ECD graft. Moreover, the timely nature of the operation and the standardized quality of the LD graft should be considered, in contrast to the emergency operation and the vast heterogeneity of ECD grafts.
Our study has some limitations that need to be adressed, the most important being its retrospective single-center design. Moreover, there was vast heterogeneity among the quality of the ECD grafts. Finally the diverging definition criteria of ECD grafts represents another considerable limitation that may affect the generalization of our results to the spectrum of different criteria used by other centers.

Despite the observed survival benefit, the risk-benefit ratio in patients receiving ECD grafts must be evaluated on a caseby-case basis according to the waiting list and the conservative versus expanded policies of each transplant center. It should also be highlighted that the diverging criteria for the definition of ECD grafts represent a considerable limitation to the wide application of this policy. Regardless, developing alternative strategies for the continued expansion of the available organ pool is critical [26]. Thorough and detailed knowledge of the different aspects of liver donation may contribute to further amelioration of donor safety and even recipient outcomes. 


\section{Summary Box}

\section{What is already known:}

- Hepatocellular carcinoma (HCC) is one of the most common malignancies worldwide, with rising incidence

- Liver transplantation (LT) is considered to be the treatment of choice for early HCC in patients with end-stage liver failure, but is limited by donor organ availability

- Live donor LT (LDLT) has become the most promising alternative for the expansion of the organ pool for adult patients with HCC, but indications and criteria are still subject to debate

- Extended criteria donors (ECD) represent a reliable alternative

\section{What the new findings are:}

- LT for HCC showed superior patient survival with ECD rather than LD grafts

- Re-LT, ischemic type bile lesions, and tumor recurrence showed no significant differences between groups

- Although LDLT for HCC showed inferior survival results in comparison to ECD-LT, the timely fashion of the operation and the standardized quality of the live donor graft should be considered, in contrast to the emergency operation and the vast heterogeneity of ECD grafts

\section{References}

1. El-Serag HB, Mason AC. Rising incidence of hepatocellular carcinoma in the United States. N Engl J Med 1999;340:745-750.

2. Kew MC. Epidemiology of hepatocellular carcinoma. Toxicology 2002;181-182:35-38.

3. Mazzaferro V, Regalia E, Doci R, et al. Liver transplantation for the treatment of small hepatocellular carcinomas in patients with cirrhosis. N Engl J Med 1996;334:693-699.

4. Kaihara S, Kiuchi T, Ueda $M$, et al. Living-donor liver transplantation for hepatocellular carcinoma. Transplantation 2003;75:S37-S40.

5. Gondolesi GE, Roayaie S, Muñoz L, et al. Adult living donor liver transplantation for patients with hepatocellular carcinoma: extending UNOS priority criteria. Ann Surg 2004;239:142-149.

6. Lo CM, Fan ST, Liu CL, Chan SC, Wong J. The role and limitation of living donor liver transplantation for hepatocellular carcinoma. Liver Transpl 2004;10:440-447.

7. Todo S, Furukawa H; Japanese Study Group on Organ Transplantation. Living donor liver transplantation for adult patients with hepatocellular carcinoma: experience in Japan. Ann Surg 2004;240:451-459; discussion 459-461.

8. Hwang S, Lee SG, Joh JW, Suh KS, Kim DG. Liver transplantation for adult patients with hepatocellular carcinoma in Korea: comparison between cadaveric donor and living donor liver transplantations. Liver Transpl 2005;11:1265-1272.

9. Malagó M, Sotiropoulos GC, Nadalin S, et al. Living donor liver transplantation for hepatocellular carcinoma: a single-center preliminary report. Liver Transpl 2006;12:934-940.

10. Hiatt JR, Carmody IC, Busuttil RW. Should we expand the criteria for hepatocellular carcinoma with living-donor liver transplantation?-no, never. J Hepatol 2005;43:573-577.

11. Broelsch CE, Frilling A, Malago M. Should we expand the criteria for liver transplantation for hepatocellular carcinoma-yes, of course! J Hepatol 2005;43:569-573.

12. Onaca N, Davis GL, Goldstein RM, Jennings LW, Klintmalm GB. Expanded criteria for liver transplantation in patients with hepatocellular carcinoma: a report from the International Registry of Hepatic Tumors in Liver Transplantation. Liver Transpl 2007;13:391-399.

13. Sotiropoulos GC, Malagó M, Molmenti E, at al. Liver transplantation for hepatocellular carcinoma in cirrhosis: is clinical tumor classification before transplantation realistic? Transplantation 2005;79:483-487.

14. Sotiropoulos GC, Paul A, Molmenti E, et al. Liver transplantation for hepatocellular carcinoma in cirrhosis within the Eurotransplant area: an additional option with "livers that nobody wants". Transplantation 2005;80:897-902.

15. Sotiropoulos GC, Lang H, Sgourakis G, et al. Liberal policy in living donor liver transplantation for hepatocellular carcinoma: lessons learned. Dig Dis Sci 2009;54:377-384.

16. Valentín-Gamazo C, Malagó M, Karliova M, et al. Experience after the evaluation of 700 potential donors for living donor liver transplantation in a single center. Liver Transpl 2004;10:1087-1096.

17. Sotiropoulos GC, Paul A, Gerling T, et al. Liver transplantation with "rescue organ offers" within the eurotransplant area: a 2-year report from the University Hospital Essen. Transplantation 2006;82:304-309.

18. Yoo HY, Patt CH, Geschwind JF, Thuluvath PJ. The outcome of liver transplantation in patients with hepatocellular carcinoma in the United States between 1988 and 2001: 5-year survival has improved significantly with time. J Clin Oncol 2003;21:4329-4335.

19. Sotiropoulos GC, Lang H, Nadalin S, et al. Liver transplantation for hepatocellular carcinoma: University Hospital Essen experience and metaanalysis of prognostic factors. J Am Coll Surg 2007;205:661-675.

20. Malagó M, Testa G, Frilling A, et al. Right living donor liver transplantation: an option for adult patients: single institution experience with 74 patients. Ann Surg 2003;238:853-862; discussion 862-863.

21. Broelsch CE, Frilling A, Malagó M. Should we expand the criteria for liver transplantation for hepatocellular carcinoma--yes, of course! J Hepatol 2005;43:569-573.

22. Malagó M, Testa G, Marcos A, et al. Ethical considerations and rationale of adult-to-adult living donor liver transplantation. Liver Transpl 2001;7:921-927.

23. Okuda K, Ohtsuki T, Obata $H$, et al. Natural history of hepatocellular carcinoma and prognosis in relation to treatment. Study of 850 patients. Cancer 1985;56:918-928.

24. Sotiropoulos GC, Tagkalos E, Fouzas I, et al. Liver transplantation for hepatocellular carcinoma using extended criteria donor grafts. Transplant Proc 2012;44:2730-2733.

25. Benko T, Gallinat A, Minor T, et al. The postoperative Model for End stage Liver Disease score as a predictor of short-term outcome after transplantation of extended criteria donor livers. Eur $J$ Gastroenterol Hepatol 2017;29:716-722.

26. Vodkin I, Kuo A. Extended criteria donors in liver transplantation. Clin Liver Dis 2017;21:289-301. 